\title{
Reanimate the Dead to Life by Practicing Cryo Techniques
}

\author{
Ashutosh Menon* \\ Bombay University, Mumbai, India \\ ${ }^{\star}$ Corresponding author: Ashutosh Menon, Bombay University, Mumbai, India; Mobile: 09136176178; Email: ashutoshkaladhar@gmail.com
}

Received: December 07, 2021; Accepted: December 21, 2021; Published: December 31, 2021

\begin{abstract}
Therapeutic Hypothermia has been evolving as a science in itself since a very long time. There have been many accidents chronicled wherein people were buried alive in snow or under ice in frigid waters, left scientifically dead without any signs of heartbeat and breathing, only to be revived many hours later. The most notable case being that of Anna Bagenholm from Sweden. Doctors in Siberia have been placing babies in snow banks since the 1960 's before surgery to increase their chances of survival. Stowaways have been known to survive in the wheel well of an aircraft jetliner at 38,000 feet altitude with negligible oxygen \& -50 degree temperatures. Inspiration is also drawn from hibernation ability of the hibernating animals like the artic ground squirrel, wood frog, nematodes, polar bears etc. in harsh winter conditions. But it has been felt that the real benefits of medical hypothermia have not been exploited so far for the progress of mankind. By means of this research, we have now discovered how cooling can assist in bringing back the dead to life.

Pigs were bled to death with a major trauma to their vital common jugular vein and/or carotid artery to mimic an accident/trauma injury. The pig had by now lost its heartbeat, pulse, blood pressure, respiration, etc. and all other vital signs of life, leaving it clinically dead. Its chest cavity was opened via a thoracotomy. A cannula or long specialised Cather was inserted into the aorta. Dead bodies of these pigs were immediately cooled by sucking out their entire blood through the aorta \& replacing it with an ice cold saline solution (at 5 degrees Celsius) suspended with PFC's - Perfluorocarbon aggressively pumped into the aorta. PFC's are efficient artificial oxygen carriers. All this drained blood of the pig was carefully collected in a vat for re-transfusion in future. This cold oxygenated solution was continuously circulated through the blood vessels of the dead pig bringing its core body temperature to about 10 degrees Celsius rapidly within 10 minutes. An ECMO machine was connected with a chiller and heater alternatively, used continuously for all circulatory purposes of both the warm blood \& ice cold solution throughout the pig's body. External cooling packs \& ice pads were also applied on the entire body of the pig to cool down its dead body as soon as possible. A surgery was performed on the dead body to restore the tear (sew up the vein which had been severed). The pig was slowly thawed in a warm water bath at about 0.5 degree Celsius per hour to the normal body temperature of 38 degrees of the pig. The ice cold saline was then replaced with the warm original blood of the pig when the temperature reached 30 degrees. After about reaching to 32 degrees some of the pig's heart started beating automatically while the others had to be given a jumpstart using external electric shocks called defibrillation or Electrical Cardioversion. The pigs were immediately connected to a ventilator device for assisted respiration. After about a day or so these pigs returned to normalcy with regards to heart \& breathing rhythms. By following the above procedure dead humans may also be brought back to life immediately after demise. This procedure will be more effective in dead youths who unfortunately got killed. Pigs were used for this experiment as they share a number of surprising comparable traits with humans which are a well-known fact.
\end{abstract}

Keywords: Cryo technique, Therapeutic hypothermia, Cooling, Suspended animation, Resurrect dead to life, Cryopreservation, Cryogenics, Emergency medicine

\section{Introduction}

The word hypothermia comes from the Greek language. Therapeutic hypothermia has been around since ancient times. It has been practiced since the past 5000 years in varying intensities. It was even used by the ancient Egyptians and finds mention in the "Edwin Smith Papyrus". Even the ancient Greek physician Hippocrates has stated some uses of cooling in medicine in accordance with the knowledge of his times. Hypothermia was back in vogue in the $17^{\text {th }}$, $18^{\text {th }} \& 19^{\text {th }}$ Centuries in different types of treatments like cold water bathing, spas etc. for general fitness \& wellbeing. Sometimes cold was used as a sedative. It was sometimes seen as an alternative medical practice also. Freezing water was poured on psychiatric patients as a method to treat mental disorders. They were tortured by forcibly dipping them in ice cold water. But in this paper we are exploring an advanced use of clinically controlled therapeutic hypothermia to help bring back the dead to life.

\section{Methods/Procedure}

The dead body can be cooled rapidly after sudden death, repaired \& thawed back slowly to life. This process is most effective in young patients when even after death occurs there is minimal damage to the body structures/physical anatomy of the human body.

As noted the brain cells begin to die within 10 minutes after respiration $\&$ heartbeat stops due to non-receipt of fresh oxygen from the blood. 
This process of brain cell death can be prevented by placing ice packs over the head immediately after death or immersing the entire head in an ice bucket or ice bag at once after death. This may be a crude technique but a very effective first aid in locations where no other medical assistance such as a Cryo Kit is unavailable. CPR is continuously kept done to circulate the blood till reaching the cryo facility either manually or by a machine available at the scene. Supportive oxygen or air should be forced into the lungs if possible. Utmost care must be taken to prevent any brain cells from dying of oxygen deprivation.

The Cooling using ice or thermal blankets reduces the possibility of the neural damage to the brain to some extent.

Once the onsite medical practitioner has confirmed death by checking the routine pulse \& breathing parameters, the cold dead body should be immediately rushed to the cryo rusticating ICU facility in a hospital.

At the Cryo facility anti blood coagulating injections like heparin are immediately injected into the cadaver. The chest cavity is opened. Then the entire blood is flushed out of the body through the aorta within a few minutes using a high pressure pump. The adult human body has just about 5 Litres of blood.

There are two kinds of processes developed to bring back the dead to life.

1. The Chilled Process at 10 degree

2. The Frozen Process at minus - 18 degree

\section{The Chilled Process at 10 Degree}

The body is transported to a chilled operation theatre temperature controlled at 10 degrees Celsius. All the blood is immediately replaced with a 0.9 concentration saline solution suspended with perfluorocarbons cooled to 5 degrees Celsius. This maintains the core temperature of the body at 10 degrees Celsius which is constantly monitored using an advanced body heat probe.

The cooled saline solution is continuously oxygenated and kept circulating in the body using an ECMO Machine. These PFC's in the solution carry oxygen to the cells \& remove the carbon dioxide.

Oxygen dissolved in saline solution is a bit less but enough for the now chilled brain cells which have no or minimal oxygen demand.

Since, $5 \%$ Metabolism of the cells falls with every degree Celsius drop in temperature. Our general body temperature is 37 degrees Celsius. Therefore, chilling the core body temperature to 10 degrees practically stops all physical, chemical \& biological processes at the cellular level of the body.

The body is thus put in a state of suspended animation which arrests all of the metabolic activities. In this state of suspended animation, the defect that caused death is rectified. For example, if death was due to a cardiac arrest the blood vessels of/near the heart are cleared. Angioplasty or bypass surgery may be performed. If the death was due to gunshot or stabbing the wound the organ damage is repaired artificially and sutured. The injuries are repaired. If the death was due to an accident, the trauma borne parts are repaired surgically. If the death was due to poisoning the poison is completely flushed out of the system etc. and so on. Death should have happened due to fixable causes.

Once the reason for death has been cured, the body is moved to room temperature \& is slowly thawed back by placing it in a water bath.

The saline solution is pumped out and replaced by warm blood when the thawing reaches 30 degrees. The heart is given a jump start to beating by electrical shock to begin beating. EPR (Electro-Pulmonary Resuscitation). Slowly the temperature is increased over a period of many hours to the normal 37 degrees@ 0.5 degrees per hour.

Once the hearts start beating back on its own the patient may be provided with a ventilator device for a few days/weeks to assist breathing. After the patient is fully recovered from whatever was his cause of death \& is declared fit \& fine, He or she can be discharged from the hospital if all the vital parameters such as BP, SPO2, ECG, EEG, BPM, etc. are back to normal.

\section{The Frozen Process at -18 degree}

The frozen process is very similar to the chilled process. The only change being that the temperature set is at minus - 18 degrees.

The body is transported to a frozen operation theatre temperature controlled at minus -18 degrees Celsius. It may be even equipped with a blast freezer to freeze the arriving dead body to -18 degrees rapidly.

All the blood is immediately replaced with a Cryoprotectant solution such as ethylene glycol, dimethyl sulfoxide (DMSO), glycerol, etc. cooled to minus 18 degrees Celsius which may or may not be suspended with PFC.

This cryoprotectant is to prevent the formation of large ice crystals within \& outside the cell structure which will pierce \& damage the cell membrane and kill the cells. It also protects the cells from dehydration that may occur by replacing the water within the cells \& damage the cellular structure.

The cryoprotectant helps flash freezing or Vitrification to - 18 degree of the body using a blast freezer without lethal ice crystal formation.

This process is mainly used to keep the dead animated for a longer period for a few days \& cure their cause of death effectively if possible.

This maintains the core temperature of the body at -18 degrees Celsius.

The cooled cryoprotectant solution is continuously oxygenated and kept circulating in the body using an ECMO Machine.

Oxygen dissolved in cryo solution is very less as compared with blood, but enough for the now frozen brain cells if required.

Since, $5 \%$ Metabolism of the cells falls with every degree Celsius drop in temperature. Our general body temperature is 37 degrees Celsius. Therefore, chilling the core body temperature to -18 degrees practically stops all physical, chemical \& biological processes at the cellular level of the body. 
The body is thus put in a state of suspended animation which arrest all of the metabolic activities. In this state of suspended animation, the defect that caused death is rectified. For example, if death was due to a cardiac arrest the blood vessels of/near the heart are cleared. If the death was due to gunshot or stabbing the wound and organ is repaired artificially and sutured. If the death was due to an accident, the trauma borne parts are repaired surgically. If the death was due to poisoning the poison is completely flushed out of the system. It can also be a bridge towards organ transplant till the matching donor organ becomes available. Special surgical equipment's may be needed to operate as the dead body is frozen stiff and is hard like stone at -18 degrees.

Once the cause of the death has been cured, the body is moved to room temperature \& is slowly thawed back by placing it in a water bath.

The cryoprotectant solution is pumped out and replaced by warm blood at 30 degrees. The heart is given a jump start to beating by electrical shock to begin beating. Patient is thawed slowly \& steadily to the normal 37 degrees @ 0.5 degrees per hour.

Once the hearts start beating back on its own the patient may be provided with a mechanical ventilator for a few days/weeks to assist breathing. After the patient is fully recovered from whatever was his cause of death \& fit \& fine. He can be discharged from the hospital if all the vital parameters are back to normal.

This procedure is less preferred over 10-degree saline solution process as cryoprotectants are toxic to some extent. Freezing also has a drawback of ice crystal formation danger. It is best if the patient is revived within a few hours after death using the 10-degree procedure. More time the dead body is kept in suspended animation, lesser are the chances of bringing it back to life as degradation still begins at a microscopic level.

This procedure may also be related to cryonics wherein the dead patient is vitrified to a glass like phase and ultimately stored in liquid nitrogen at -196 degrees with a vision to resurrect them someday in the future. However, no dead cryonic patient is yet known to be successfully reanimated till date. Thus if this procedure is used then the chances of bringing back the dead body back again to life is minimal, taking into account the technology available today.

\section{The PIG Experiment}

A pig has been used as a subject for this experiment. All the blood from the live pig as pumped out from a major artery (aorta), leaving the pig without any heartbeat or respiration. Inducing a cardiac arrest, a vital vein (jugular vein) on the pig was also cut to mimic an external trauma. Basically an uncontrolled lethal haemorrhage. Essentially the definition for clinical death.

Then the pigs dead body was constantly circulated with oxygenated saline solution /electrolyte solution cooled at 10 degrees Celsius. PH around 5.5. This solution can be reinforced by perfluorocarbons (PFC are artificial oxygen carriers) or RBC'S - Red Blood Cells of the correct blood group suspended in it to maximize its oxygen carrying capacity. The chest cavity is opened. The cold solution is pumped into the aorta. Arteries leading downward in the body are clamped to make sure that the brain \& heart get this cold solution first. Then this clamp is removed allowing the full body to receive the cold solution. At this point the body will have no pulse, no blood pressure, no heartbeat, no respiration \& no brain activity. That is the clinical definition of death. The cut major vein was sown up \& repaired through which the saline solution /blood loss was bound to happen.

After thawing the pig back slowly to 38 degrees at the rate of 0.5 degree per hour, to prevent any reperfusion injury the heart may start beating again on its own. Or the heart can be jump started with electrical shocks, the pigs fully recovered from clinical death. The pig may require assisted breathing or a ventilator for a few days to normalize heart \& respiration rhythms.

The pigs were earlier trained to identify different colours \& smells, prior to death, they retained the same ability \& memory even after resuscitation. This proves that there was no major brain damage or neurological impairments sustained because of this procedure.

\section{Results}

The results were quite encouraging. On an average $90 \%$ i.e. 9 out of 10 pigs survived this gruelling procedure. It is observed that, time can be brought to a halt for the patients for a few hours with use of extreme hypothermia and all activities of the body at the cellular level freezes and can be made to come to a standstill. They can be basically frozen in time.

Using these results as a blueprint dead humans can be immediately cooled rapidly after death preserving their cellular structure intact and be gradually thawed back to life after fixing their reason for death. If the cause of death was a major blood loss due to a traumatic injury or accident, then blood transfusion can be done to recompense for this lost blood.

This experiment on being performed on farm animals, especially pigs, as the anatomy of pigs is similar to humans. If the brain cells of human beings remain intact to some extent by use of low temperatures, they can be revived with no or minimal neurological damage. No memory would be lost $\&$ there would be no brain impartments.

This technique should be actively implemented mainly on young patients who unfortunately die early from repairable causes, to grant them a second chance at life. These results can act as a small boost in the field of cryonics freezing patients in liquid nitrogen at -196 degrees or freezing patients for inter galactic travel in future. But this is just a baby step, and it may have many other encouraging future applications also.

\section{Discussions}

Apparatus required in the emergency Cryo ICU are:

1. Temperature Controlled ICU Chamber at 10 degrees with or without a blast freezer.

2. 0.9 Concentration $\mathrm{NaCl}$ Sodium Chloride Saline solution which may be suspended with at least $20 \%$ perfluorocarbons.

3. AV Artery Venous ECMO Device (Extracorporeal membrane oxygenation) or Heart Lung Machine with attached pump to perform the functions of both the heart \& lungs. 
4. Cooler, Heat Exchanger \& Condenser System etc. connected to the ECMO to keep the circulating saline solution at 10 degrees continuously.

5. IV - Intravenous Cannulas to insert directly into the aorta to pump the blood out \& cold solution in and out rapidly.

6. Water bath which can be increased to a temperature of 37 degrees in another chamber to increase the body temperature slowly to avoid any reperfusion injury.

Warmed blood, the body should be thawed slowly at a rate of 0.5 degree per hour. (medium thawing) Trained personal in Cryo Cooling Science.

Apparatus required in the Emergency Mobile Cryo Ambulance:

1. Cooling Pads or Cooling bag filled with with cold gel or circulating cold fluids to cover \& cool the entire dead body, especially the head immediately.

2. Automated chest compression machine to perform CPR to maintain the blood circulation.

3. Supplementary Oxygen from cylinder to provide artificial respiration.

4. Anti-blood clotting injections such as Heparin.

The patient must be reached as soon as possible to the Cryo ICU at the hospital after on site stabilisation by the paramedics.

Apparatus in Cryo First Aid Kit:

1. Lots of Ice to at least cover the head of the dead body. The more part of the dead body covered with ice the better. This will slow down organ damage.

2. CPR to be performed manually.

3. Artificial mouth to mouth breathing to be given every $7-10$ seconds.

It is very important to begin all these procedures immediately after death on the cadaver.

More the time lost before therapeutic cooling less are the chances of resurrecting the dead patient. At most a dead patient can be resurrected 1 hour after death in normal circumstances. Because brain cell death \& neurological damage begins within 10 minutes the body stops receiving oxygen. Other vital organs \& tissues also suffer from oxygen deprivation at normal temperatures. As time is lost, even after bringing back the dead body to life, the patient may have to live lifelong with irreversible brain damage. It is almost impossible to bring back to life someone who has been dead and warm for 10-12 hours. As the old saying goes "You are not dead until warm \& dead".

Bringing back the dead to life using cryo technique is more suitable for young dead bodies under the age of 60 years. This is because their systems are generally more robust to handle the strain of this procedure. This procedure is not efficient in people with terminal incurable illness like end stage cancer or HIV/AIDS as they are deemed to die again soon even if brought back to life by using this procedure temporarily. Therefore, a do not rusticate code may be practiced with such patients.

\section{Conclusion}

From the above research it may be concluded that, immediate cooling especially of the head, should be applied as a standard operating procedure at once after death is confirmed.

This cooling may be performed by any frozen material available at hand on site such as ice, cold water bags or even frozen food packets until the paramedics arrive. Even a Cryo Cooling Machine which can double up as a common food refrigerator when not in use may be purchased \& kept handy for this purpose in every household \& commercial establishment.

Every ambulance must be compulsorily equipped with the above suggested basic cryo cooling kit, to safely regulate the dead patients' temperature until reaching the Cryo ICU at the earliest.

At the Cryo ICU the trained team of doctors, surgeons, super specialists, perfusionists, nurses, etc. should revive the dead patient using the most advanced technology as stated in this article \& reverse death.

This procedure of Cryo Resurrection should be strictly adopted at all major medical facilities especially on the unfortunate young dead patients. In this way we can give the dead a second chance by bringing them back to life.

\section{Declaration of Conflicting Interest}

The author declares that there is no conflict of interest.

\section{Funding}

Due to lack of Funds, this research project could not be completed \& had to be abandoned.

This research received no specific grant from any funding agency in the public, commercial, or not-for-profit sectors.

\section{Acknowledgements}

I would like to take this opportunity to thank my mother Mrs Sathi Devi Menon who is always by my side rock solid and is also my biggest supporter. To my father Mr KRK Menon who struggled for years to provide the best education to me as a child. Without their unrelenting cooperation \& support this research would have never been possible [1-7].

\section{Reference}

1. Samuel A. Tisherman. Emergency Preservation and Resuscitation for cardiac arrest from trauma (EPR-CAT). University of Maryland School of Medicine. [crossref]

2. Dr Phil Jaekl. Curiosities of Medical History: The controversy of using cold as a treatment - Medical News today.

3. Hasan BA. Learning and memory is preserved after induced asanguineous hyperkalemic hypothermic arrest in a swine model of traumatic exsanguination. [crossref]

4. Safar P, Brown T, Holtey W (1961) Ventilation and circulation with closed chest cardiac massage in man. JAMA 176: 574-576 [crossref] 
5. Rhee PM, Acosta J, Bridgeman A, Wang D, Jordan M, et al. (2000) Survival after emergency department thoracotomy: review of published data from the past 25 years. Journal of the American College of Surgeons 190: 288-298. [crossref]

6. Samuel AT, Hasan BA, Peter MR, Thomas MS, Tomas D et al. Development of the emergency preservation and resuscitation for cardiac arrest from trauma clinical trial. [crossref]

7. Peter Rhee (2000) Induced Hypothermia during Emergency Department Thoracotomy: An Animal Model. The Journal of trauma 48: 439-447. 